OPEN ACCESS

Edited by:

Enrico Baruffini,

University of Parma, Italy

Reviewed by:

Ken Lieuw,

Uniformed Services University

of the Health Sciences, USA

Max Christoph Liebau,

University Hospital of Cologne,

Germany

*Correspondence:

Eleanor G. Seaby

e.seaby@soton.ac.uk

Specialty section:

This article was submitted

to Genetic Disorders,

a section of the journal

Frontiers in Pediatrics

Received: 21 February 2017

Accepted: 01 May 2017

Published: 22 May 2017

Citation:

Seaby EG, Gilbert RD, Andreoletti G,

Pengelly RJ, Mercer $C$, Hunt $D$ and

Ennis S (2017) Unexpected Findings in a Child with Atypical Hemolytic

Uremic Syndrome: An Example of

How Genomics Is Changing the

Clinical Diagnostic Paradigm.

Front. Pediatr. 5:113.

doi: 10.3389/fped.2017.00113

\section{Unexpected Findings in a Child with Atypical Hemolytic Uremic Syndrome: An Example of How Genomics Is Changing the Clinical Diagnostic Paradigm}

\author{
Eleanor G. Seaby ${ }^{*}$, Rodney D. Gilbert ${ }^{2,3}$, Gaia Andreoletti ${ }^{1}$, Reuben J. Pengelly', \\ Catherine Mercer ${ }^{4}$, David Hunt ${ }^{4}$ and Sarah Ennis ${ }^{1}$ \\ ${ }^{1}$ Human Genetics and Genomic Medicine, Faculty of Medicine, University of Southampton, Southampton, UK, \\ ${ }^{2}$ Wessex Regional Paediatric Nephro-Urology Service, Southampton Children's Hospital, Southampton, UK, \\ ${ }^{3}$ Faculty of Medicine, University of Southampton, Southampton, UK, ${ }^{4}$ Wessex Clinical Genetics Service, \\ Princess Anne Hospital, Southampton, UK
}

$C B L$ is a tumor suppressor gene on chromosome 11 encoding a multivalent adaptor protein with E3 ubiquitin ligase activity. Germline CBL mutations are dominant. Pathogenic de novo mutations result in a phenotype that overlaps Noonan syndrome (1). Some patients with CBL mutations go on to develop juvenile myelomonocytic leukemia (JMML), an aggressive malignancy that usually necessitates bone marrow transplantation. Using whole exome sequencing methods, we identified a known mutation in CBL in a 4-year-old Caucasian boy with atypical hemolytic uremic syndrome, moyamoya phenomenon, and dysmorphology consistent with a mild Noonan-like phenotype. Exome data revealed loss of heterozygosity across chromosome $11 \mathrm{q}$ consistent with JMML but in the absence of clinical leukemia. Our finding challenges conventional clinical diagnostics since we have identified a pathogenic variant in the CBL gene previously only ascertained in children presenting with leukemia. The increasing affordability of expansive sequencing is likely to increase the scope of clinical profiles observed for previously identified pathogenic variants and calls into question the interpretability and indications for clinical management.

Keywords: aHUS, genomics, JMML, preclinical cancer, whole-exome sequencing

\section{CASE PRESENTATION}

Our patient presented aged 4 months with right-sided focal seizures. He had been born at term following an uncomplicated pregnancy. A brain magnetic resonance imaging (MRI) scan revealed a left cerebral artery infarct and occlusion of the left internal carotid artery with collateral flow in keeping with moyamoya phenomenon; sequelae have included a right hemiparesis and dysarthria.

At the age of 2 years, this patient had marked thrombocytopenia (platelets $26 \times 10^{9} / \mathrm{L}$, hemoglobin $115 \mathrm{~g} / \mathrm{L}$ ), mild proteinuria (urine protein/creatinine ratio $42 \mathrm{mg} / \mathrm{mmol}$ ), and hypertension. Investigations revealed normal range renin, aldosterone, reticulocyte count, lactate dehydrogenase, von Willebrand factor, and ADAMTS13. He had a negative Coombs test, but low serum complement 
C3 (0.59 g/L, normal 0.75-1.65). Complement C4 was normal (0.14 g/L, normal 0.14-0.54). Alternative pathway hemolytic complement activity was low at 28\% (normal $80-200 \%$ ) but total hemolytic complement was normal at $92 \%$, suggesting dysregulated activation of the alternative complement pathway. Red cell fragments were absent on blood film but the haptoglobin concentration was reduced at $0.17 \mathrm{~g} / \mathrm{L}$ (normal 0.5-2.0). A karyotype was normal. Renal biopsy was unremarkable by light microscopy apart from light C3 staining along capillary walls. Electron microscopy confirmed endothelial cell separation from the glomerular basement membrane with accumulation of fluffy subendothelial material consistent with endothelial damage. Atypical hemolytic uremic syndrome was considered likely and possibly the cause of his cerebral infarct; he thus commenced eculizumab therapy. Sequencing of the coding regions and flanking sequences of $C 3, C F I, C F B, C D 46$, and DGKE revealed no pathogenic mutations. Sequencing of $C F H$ revealed a heterozygous variant (c.G2850T:p.Q950H), which at the time was of unknown clinical significance.

Examination at age 3 revealed dysmorphic features (Figure 1). His spleen was palpable $5 \mathrm{~cm}$ below the costal margin, and he had a right-sided hemiparesis with upper arm withdrawal reflex and down-going plantars. He had marked dysarthria and only spoke single words. Developmentally, he had skills appropriate for a $1 \frac{1 / 2}{2}-2 \frac{1}{2}$-year old, which were attributed to his cerebral infarct. He had previously undergone orchidopexy to correct bilateral cryptorchidism. Despite an improvement in his platelet count following continued eculizumab therapy, he remained variably thrombocytopenic (platelet counts $85-181 \times 10^{9} / \mathrm{L}$ ) with marked splenomegaly. A bone marrow aspirate showed no impaired

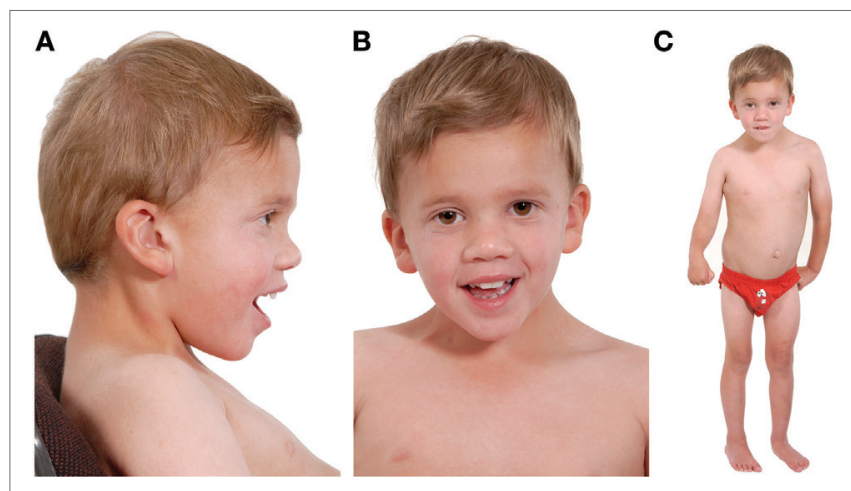

FIGURE 1 | Three photographs of our patient taken at the age of 4 years. Photograph (A) shows a low posterior hairline with low-set posteriorly rotated ears, microcephaly, and mild frontal bossing. Photograph (B) shows down-slanting palpebral fissures and mild ptosis. He has dental crowding and a narrow, high-arched palate (not shown). Photograph (C) shows lasting damage from his cerebral infarct: the right upper limb is flexed and there is reduced muscle tone of the thigh in comparison to the left lower limb. His neck appears broad, with a broad thorax and wide-spaced nipples. His spleen measures $12 \mathrm{~cm}$ and marginally distorts the appearance of the abdomen. His skin is soft and mottled and he has clinodactyly. He is $<0.4$ th centile for height, weight, and head circumference, and developmentally he is predicted to have skills appropriate for a $2 \frac{1}{2}-3$-year old. Noteworthy, during the antenatal period, marginal nuchal fold enlargement was documented but otherwise pregnancy and delivery were uneventful. thrombocyte production or morphological abnormalities; therefore, his thrombocytopenia was attributed to hypersplenism.

The clinical hypothesis was an endothelial abnormality which interfered with complement regulation, possibly by reducing factor $\mathrm{H}$ binding, causing thrombotic microangiopathy (TMA) involving the kidneys and brain. The splenomegaly was not explained.

\section{MATERIALS AND METHODS}

WES was undertaken in an attempt to elucidate the pathophysiology. Genomic DNA was extracted from whole blood, and target capture was performed on Agilent's SureSelect v5.0 (51 Mb). The enriched library was sequenced on the Illumina HiSeq2000. The identity and provenance of returned sequencing data were validated through application of an optimized genotyping panel (2). WES data were analyzed using an in-house pipeline as previously described $(3,4)$. Candidate genes were selected using curated databases of pathogenic variants associated with search terms applicable to the phenotype of interest.

\section{RESULTS}

In total, 24,955 variants were called with an average read depth of $58 \times$. Of these, 470 variants were loss of function mutations, 2,631 were splicing variants, 11,146 were synonymous, and 10,708 were non-synonymous single nucleotide variants. Primary analysis comprised filtering on a targeted panel of 540 complementassociated genes; 916 variants were called. Variant prioritization identified two variants of unknown significance: the same variant in $C F H$ (c.G2850T:p.Q950H) as found in the aHUS gene panel and a splicing variant in $C R 1(\mathrm{c} .7252+1 \mathrm{G}>\mathrm{A})$. The results were equivocal.

Two years later, this case was revisited following clinical review. Phenotypic information concerning moderate splenomegaly, persistent thrombocytopenia (despite eculizumab therapy, a normal bone marrow aspirate, and unchanged appearances of the cerebral magnetic resonance angiography) informed a revised analysis of further 44 genes collated from a literature search of PUBMED and the Human Gene Mutation Database using the search terms thrombocytopaenia, splenomegaly, and moyamoya. Filtering parameters reduced 51 variants to one heterozygous splicing variant in $C B L$ (c.1096-1G $>\mathrm{T}$ ) predicted to be pathogenic following application of the American College of Genetics and Genomics guidance (5). The mutation was validated by Sanger sequencing and segregation analysis confirmed de novo inheritance following the absence of the splicing mutation in both parents (Figure 2).

In children harboring germline $C B L$ mutations, juvenile myelomonocytic leukemia (JMML) usually develops following somatic loss of heterozygosity (LOH) of chromosome 11q (6) although some patients develop JMML without LOH. In nearly all cases, the mutant allele is duplicated by acquired uniparental isodisomy, resulting in loss of the wild-type tumor suppressor allele and duplication of the oncogenic mutation (Figure 3) (1, $7,8)$. WES data were, therefore, scrutinized for $\mathrm{LOH}$ across chromosome 11 in the region of the $C B L$ locus; this was facilitated 


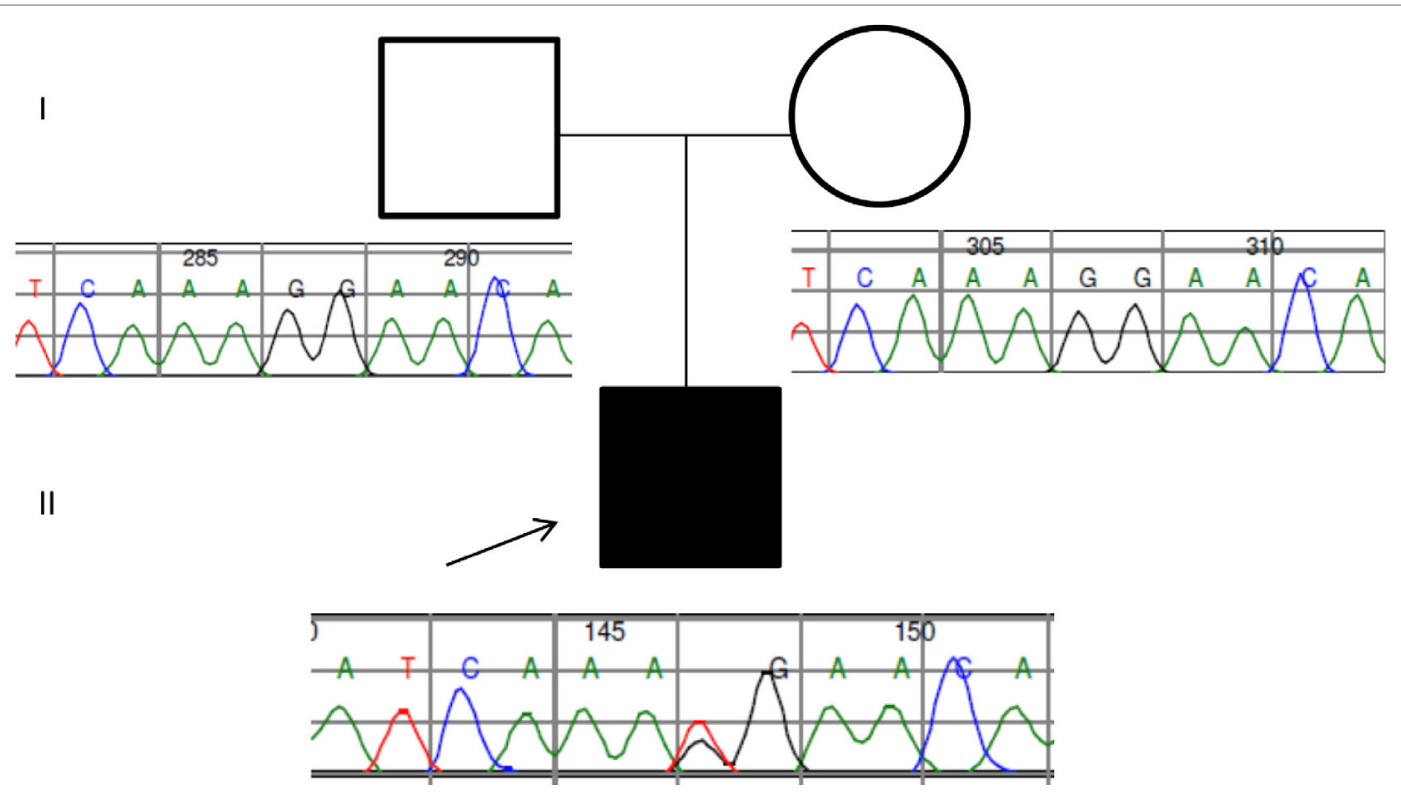

FIGURE 2 | Pedigree and Sanger traces of the patient/parent trio. The proband (filled black square) has a de novo CBL heterozygous mutation of c.1096-1G>T, affecting the canonical splice site.

by peripheral blood-derived DNA. We retrospectively assessed $\mathrm{LOH}$ by plotting $\mathrm{B}$-allele frequency ratios across the exome (Figure S1 in Supplementary Material) (9). On average, 70\% of the sequenced reads mapping across chromosome 11q harbored the mutant allele compared with the reference. This significant allelic imbalance strongly suggested a clonal advantage of (a subset of) peripheral leukocytes consistent with myeloproliferation and a potential transformation to $\operatorname{JMML}(6,7)$.

The two variants of unknown significance in CR1 and $\mathrm{CFH}$ were also revisited. Sanger sequencing confirmed both heterozygous variants in the proband. Neither variant was de novo; the $C F H$ variant was inherited from the proband's asymptomatic father, and the $C R 1$ variant was inherited from his asymptomatic mother. To assess functional significance, red blood cells (RBCs), plasma and DNA were sent to the Jokiranta Research Group, at the University of Helsinki for functional analysis. CR1 was ruled out as pathogenic following normal expression of the complement receptor 1 on erythrocytes with normal levels of C3dg on the RBC surface. The Q950H variant of Factor $\mathrm{H}$ is now known to impair factor $\mathrm{H}$ activity (10), providing a genetic explanation for the patient's aHUS. As previously reported, most aHUS mutations show variable penetrance, providing explanation for why the proband's father is unaffected (11).

\section{DISCUSSION}

Germline $C B L$ mutations have been associated with Noonan-like syndrome, moyamoya, and vasculitis (12). Our patient displays many of the phenotypic features consistent with germline $C B L$ mutations. Although his global developmental delay had been attributed to his cerebral infarct, review of his MRI scans suggests topographical inconsistencies; therefore, in retrospect, it is likely that a proportion of his developmental delay results from his $C B L$ mutation.

Juvenile myelomonocytic leukemia is an aggressive, childhood myeloproliferative disease cured only by hematopoietic stem cell transplant (HSCT), yet hematological heterogeneity has been reported; some hematological abnormalities spontaneously resolve, while others progress aggressively $(6,13)$. Data analysis poses a difficult diagnostic dilemma; our patient is displaying evidence of myeloproliferation and subclinical JMML (by discovery of clonal expansion within the peripheral blood leukocyte population) despite not manifesting clinical leukemia; he has a normal peripheral blood film, white cell count, lactate dehydrogenase level, and no increase in peripheral blood monocytes. However, his monocytes were persistently elevated between 2011 and 2014, and he continues to have a borderline monocytosis. Furthermore, he has unremitting splenomegaly, a classical feature of JMML and has intermittent, mild anemia (hemoglobin concentrations 104-124 g/L) and thrombocytopenia. Interestingly, the proband's thrombocytopenia did not fully resolve with eculizumab, potentially demonstrating marrow replacement by malignant cells or marrow failure; a common feature of JMML. This patient does not meet full hematological JMML diagnostic criteria, although he does meet oncogenetic diagnostic parameters (13) and would have met the diagnosis between 2011 and 2014 during which time he had persistent monocytosis. Even so, not all patients with $C B L$ mutations and associated LOH will develop fulminant JMML that necessitates HSCT. CBL-associated JMML does not always follow an aggressive course and can spontaneously resolve without treatment (13). That said, in previous cases of $C B L$ mutations involving the same splice site, the disease presented aggressively (6); indeed in one child, the mutation remained heterozygous in hematopoietic cells without evidence of $\mathrm{LOH}$, yet the patient still required HSCT (14). Therefore, this finding poses a challenging 


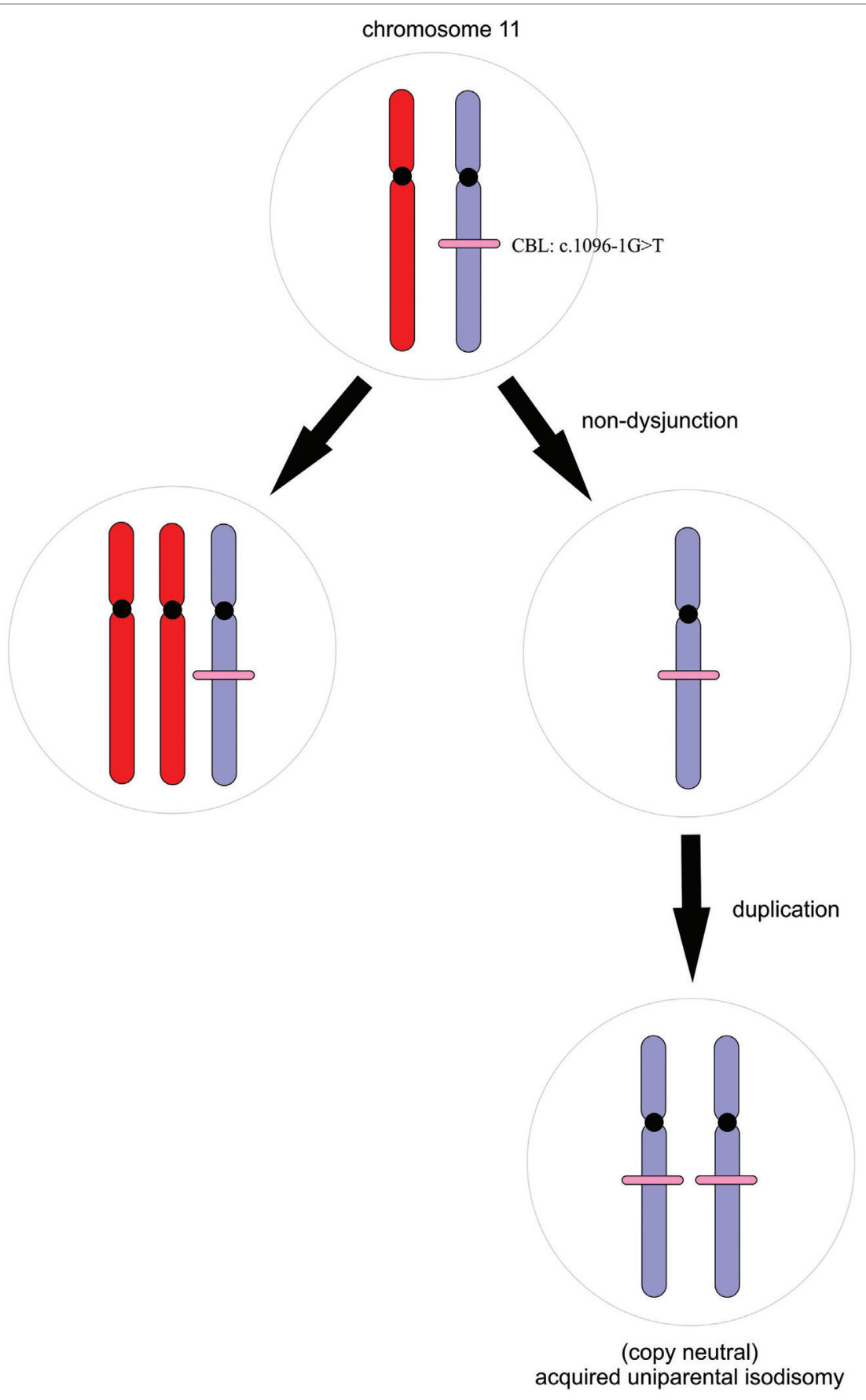

FIGURE 3 | A simple schematic illustrating the process of acquired uniparental isodisomy. For simplicity, we illustrate complete chromosomal loss of heterozygosity. Non-dysjunction results in unequal chromosomal division during mitosis. Copy neutral loss of heterozygosity occurs with duplication of the mutant allele and loss of the wild-type without a change in copy number.

clinical scenario, particularly since there is uncertainty regarding the disease trajectory.

Two differential prognoses are as follows: (a) progression to an aggressive JMML if no potentially curative HSCT intervention is offered or (b) spontaneous regression (if not already regressed) of a relatively quiescent myeloproliferative cell population that appears aggressive in the literature due to ascertainment bias. Ultimately, the biggest challenge concerns the appropriate action(s) to take, especially since a decision to proceed to transplant is not without significant risk, and there is established phenotypic heterogeneity and variable expressivity among individuals with identical $C B L$ mutations (15). The current recommendation for JMML secondary to $C B L$ mutations is that of careful surveillance. Locatelli and Niemeyer recommend that in children harboring $C B L$ mutations, 


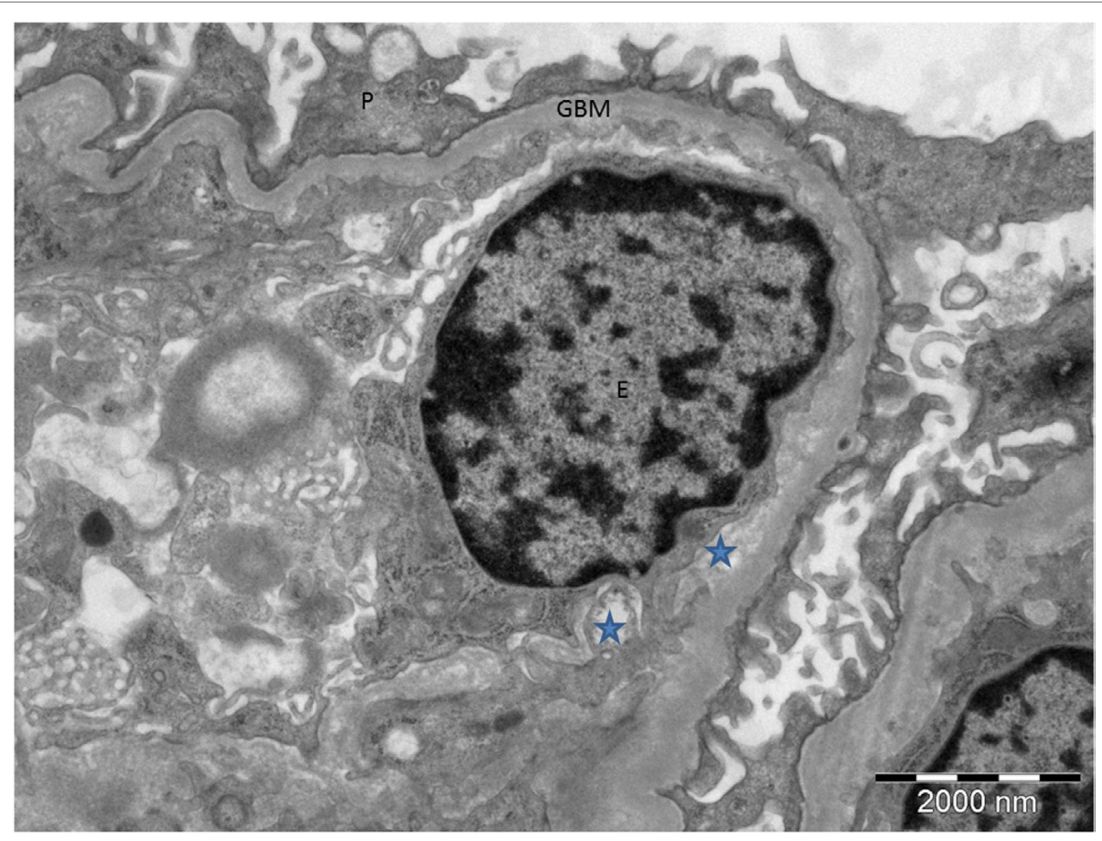

FIGURE 4 | Electron microscopy of the proband's renal biopsy demonstrating tissue microangiopathy. The stars show the subendothelial space, representing detachment of the endothelial cell from the basement membrane. P, podocyte; GBM, glomerular basement membrane; E, endothelial cell.

the decision to proceed to transplantation should be carefully weighed. They recommend adopting a "watch and wait" approach with close follow-up to enable prompt diagnosis and subsequent action should the disease evolve (13). There is, however, speculation that HSCT may prevent further vascular complications, although this is yet to be definitively proved. HSCT recipients with JMML secondary to $C B L$ mutations tend not to have further vasculitis, suggesting that these mutations cause endothelial damage (13). It seems likely, therefore, that abnormal endothelium combined with reduced complement factor $\mathrm{H}$ function may have "primed" the endothelium for complement mediated damage, resulting in TMA as shown on the renal biopsy (Figure 4). In view of the severity of his previous infarct and extensive changes in intracranial vessels on magnetic resonance angiography, there may be some justification in considering HSCT in this specific case, especially should a conservative approach result in further neurovascular or nephrological damage. However, this should be balanced against the risk of TMA following bone marrow transplantation in which the role of eculizumab is not well established.

This case also raises ethical questions with regard to return of information. There are concerns that informed consent for WES is insufficient in educating patients about the scope of potential results identified (16). When presented with data from an entire exome, there is always the possibility of incidentally discovering pathogenic mutations unrelated to the presenting phenotype (17). Although this was not an incidental finding per se, his pretest diagnosis was aHUS with atypical features. The exome analysis was primarily to elucidate a cause for his aHUS, since at the time of referral, the dysmorphology was not extensively documented and was presumed to be an unusual manifestation of TMA. His developmental delay had not been considered as independent to his infarct, and his only active clinical input was by the nephrology service. Revisiting exome data allowed for the discovery of pre-leukemia in addition to a monogenic explanation for his dysmorphology, moyamoya, and developmental delay. Relaying this information to his family necessitated reflection on how "informed" the consent truly was, as well as the magnitude, distress, and implications of finding "more than we bargained for."

Our finding lies at the nexus of genomic and clinical, hematological, nephrological, and neurological diagnostics, necessitating a multidisciplinary convergence of genomic informaticians and clinicians in discussions concerning best practice. Since there is a relative dearth of evidence concerning children who harbor $C B L$ mutations in the same splice site as our patient, and who do not progress to JMML, this paper attempts to highlight the variable expressivity of these mutations. We demonstrate how continued clinical review can inform a revised analysis of exome data and uncover unexpected findings that, in retrospect, highlight the limitations of biased and fallible clinical diagnostics. The ability to return to unbiased exome data without cost duplication is of huge diagnostic value, especially since there is no restriction to the number of times data can be revisited; for example, if the phenotype changed in the future or if functional studies reassigned a previously curated variant of unknown significance as a pathogenic allele. The full potential and utility of genomic data within the clinical setting are yet to be fully appreciated, but in the emerging genomics era, cases such as these will become increasingly prevalent, and the interpretation and translation of genomic data within clinical medicine has the potential to force a paradigm shift in clinical diagnostics with substantial prognostic impact. 


\section{AUTHOR CONTRIBUTIONS}

ES: exomic data analysis, clinical data review, phenotyping, and initial draft manuscript. RG: clinical consultant, reviewed and revised the manuscript, approved the final manuscript, and supervision. GA: bioinformatics quality control analysis, exome data analysis, and approved the final manuscript. RP: exome data analysis, reviewed the manuscript, and approved the final manuscript. CM: clinical genetics consultant, documented phenotype, and approved the final manuscript. DH: genetics specialist trainee, documented phenotype, and approved the final manuscript. SE: exomic analysis oversight, reviewed and revised the manuscript, supervision, and approved the final manuscript.

\section{ACKNOWLEDGMENTS}

Professor Zakari Jokiranta at the Jokiranta Research Group, University of Helsinki, Finland, for functional analysis of CR1

\section{REFERENCES}

1. Martinelli S, De Luca A, Stellacci E, Rossi C, Checquolo S, Lepri F, et al. Heterozygous germline mutations in the CBL tumor-suppressor gene cause a Noonan syndrome-like phenotype. Am J Hum Genet (2010) 87(2):250-7. doi:10.1016/j.ajhg.2010.06.015

2. Pengelly RJ, Gibson J, Andreoletti G, Collins A, Mattocks CJ, Ennis S. A SNP profiling panel for sample tracking in whole-exome sequencing studies. Genome Med (2013) 5(9):89. doi:10.1186/gm492

3. Christodoulou K, Wiskin AE, Gibson J, Tapper W, Willis C, Afzal NA, et al. Next generation exome sequencing of paediatric inflammatory bowel disease patients identifies rare and novel variants in candidate genes. Gut (2013) 62(7):977-84. doi:10.1136/gutjnl-2011-301833

4. Tapper WJ, Foulds N, Cross NC, Aranaz P, Score J, Hidalgo-Curtis C, et al. Megalencephaly syndromes: exome pipeline strategies for detecting lowlevel mosaic mutations. PLoS One (2014) 9(1):e86940. doi:10.1371/journal. pone. 0086940

5. Richards S, Aziz N, Bale S, Bick D, Das S, Gastier-Foster J, et al. Standards and guidelines for the interpretation of sequence variants: a joint consensus recommendation of the American College of Medical Genetics and Genomics and the Association for Molecular Pathology. Genet Med (2015) 17:405-24. doi:10.1038/gim.2015.30

6. Niemeyer CM, Kang MW, Shin DH, Furlan I, Erlacher M, Bunin NJ, et al. Germline CBL mutations cause developmental abnormalities and predispose to juvenile myelomonocytic leukemia. Nat Genet (2010) 42(9):794-800. doi:10.1038/ng.641

7. Grand FH, Hidalgo-Curtis CE, Ernst T, Zoi K, Zoi C, McGuire C, et al. Frequent CBL mutations associated with 11q acquired uniparental disomy in myeloproliferative neoplasms. Blood (2009) 113(24):6182-92. doi:10.1182/ blood-2008-12-194548

8. Chang T, Dvorak C, Loh M. Bedside to bench in juvenile myelomonocytic leukemia: insights into leukemogenesis from a rare pediatric leukemia. Blood (2014) 124(16):2487-97. doi:10.1182/blood-2014-03-300319

9. Staaf J, Lindgren D, Vallon-Christersson J, Isaksson A, Göransson H, Juliusson G, et al. Segmentation-based detection of allelic imbalance and loss-ofheterozygosity in cancer cells using whole genome SNP arrays. Genome Biol (2008) 9(9):R136. doi:10.1186/gb-2008-9-9-r136

10. Mohlin FC, Nilsson SC, Levart TK, Golubovic E, Rusai K, Müller-Sacherer T, et al. Functional characterization of two novel non-synonymous alterations and $\mathrm{CFH}$ on blood samples and DNA sent from the UK. Nikki Graham in the DNA lab in Southampton for extracting the DNA samples. David Bunyan in the Wessex Regional Genetics Centre, Salisbury, for confirmatory Sanger sequencing.

\section{SUPPLEMENTARY MATERIAL}

The Supplementary Material for this article can be found online at http://journal.frontiersin.org/article/10.3389/fped.2017.00113/ full\#supplementary-material.

FIGURE S1 | Segmented B allele frequency demonstrating significant loss of heterozygosity (LOH) on chromosome 11q. The $y$-axis denotes the proportion of alternative (B) to reference (A) alleles across all called heterozygous variants. Heterozygous calls are expected to harbor $50 \%$ of the A allele and $50 \%$ of the $\mathrm{B}$ allele (one maternal and one paternal copy). A significant perturbance in this ratio (allelic imbalance) indicates $\mathrm{LOH}$. Red boxes denote regions of $\mathrm{LOH}$ across chromosome 11q, and the green line shows segmented average of $B$ allele frequency. Preliminary copy number variant analysis of chromosome 11 is consistent with (copy neutral) acquired uniparental isodisomy.

in $\mathrm{CD} 46$ and a $\mathrm{Q} 950 \mathrm{H}$ change in factor $\mathrm{H}$ found in atypical hemolytic uremic syndrome patients. Mol Immunol (2015) 65(2):367-76. doi:10.1016/j. molimm.2015.02.013

11. Bresin E, Rurali E, Caprioli J, Sanchez-Corral P, Fremeaux-Bacchi V, Rodriguez de Cordoba S, et al. Combined complement gene mutations in atypical hemolytic uremic syndrome influence clinical phenotype. J Am Soc Nephrol (2013) 24(3):475-86. doi:10.1681/ASN.2012090884

12. Hyakuna N, Muramatsu H, Higa T, Chinen Y, Wang X, Kojima S. Germline mutation of CBL is associated with moyamoya disease in a child with juvenile myelomonocytic leukemia and Noonan syndrome-like disorder. Pediatr Blood Cancer (2014) 62:542-4. doi:10.1002/pbc.25271

13. Locatelli F, Niemeyer CM. How I treat juvenile myelomonocytic leukemia (JMML). Blood (2015) 125:1083-90. doi:10.1182/blood-2014-08-550483

14. Strullu M, Caye A, Cassinat B, Fenneteau O, Touzot F, Blauwblomme T, et al. In hematopoietic cells with a germline mutation of CBL, loss of heterozygosity is not a signature of juvenile myelo-monocytic leukemia. Leukemia (2013) 27(12):2404. doi:10.1038/leu.2013.203

15. Pathak A, Pemov A, McMaster ML, Dewan R, Ravichandran S, Pak E, et al. Juvenile myelomonocytic leukemia due to a germline CBL Y371C mutation: 35-year follow-up of a large family. Hum Genet (2015) 134(7):775-87. doi:10.1007/s00439-015-1550-9

16. McGuire AL, Joffe S, Koenig BA, Biesecker BB, McCullough LB, BlumenthalBarby JS, et al. Ethics and genomic incidental findings. Science (2013) 340(6136):1047. doi:10.1126/science. 1240156

17. Green RC, Berg JS, Grody WW, Kalia SS, Korf BR, Martin CL, et al. ACMG recommendations for reporting of incidental findings in clinical exome and genome sequencing. Genet Med (2013) 15(7):565-74. doi:10.1038/ $\operatorname{gim} .2013 .73$

Conflict of Interest Statement: The authors declare that the research was conducted in the absence of any commercial or financial relationships that could be construed as a potential conflict of interest.

Copyright (๑) 2017 Seaby, Gilbert, Andreoletti, Pengelly, Mercer, Hunt and Ennis. This is an open-access article distributed under the terms of the Creative Commons Attribution License (CC BY). The use, distribution or reproduction in other forums is permitted, provided the original author $(s)$ or licensor are credited and that the original publication in this journal is cited, in accordance with accepted academic practice. No use, distribution or reproduction is permitted which does not comply with these terms. 